\title{
ESTIMULACIÓN OPORTUNA: VENTAJAS PARA EL DESARROLLO DE SU HIJO
}

\author{
MSc. Natalia Salas Guzmán* \\ M.PI. Maria Ester Morales Ramírez**
}

La estimulación oportuna tiene como principio que "cuanto antes se aprende, mejor se aprende y más provecho se obtiene en el futuro" (Bravo y Pons, año 1, No. 5, p.66).

La familia, como ente social primario, cumple un rol fundamental en la formación y. desarrollo de la personalidad de los miembros que la conforman, y se presenta además como el primer ámbito donde se estimula y educa al niñol niña, por lo tanto, la estimulación oportuna empieza en el hogar.

El presente articulo hace referencia a la estimulación oportuna, como objetivo primordial para el crecimiento y educación, estimulando los cinco sentidos, y marcando la importancia del papel de los padres como enies altamente significativos para el adecuado desarrollo integral de la personalidad de los niños / niñas, en sus áreas física, cognitiva, lingiuística, psicológica y social.

\begin{abstract}
Early stimulation presents as it's principle that "the earlier the child leams, better profits and more advantages he/she will obtain from the future" (Bravo y Pons, año 1, No. 5, p.66).

The family: as a unique and first encounter for the child, supplies a fundamental role in the structure and development of the child's personalit; presenting it self as the main environment where the child is educated and stimulated, therefore, early stimulation starts at home.

The present article establishes the importance of early stimulation in the child's objectives for life, in it's developmental and educational compounds, showing the five senses in which it can be worked, and explaining the highly significant role that the parent has in the integral development of the personality in the child, in it's physical. cognitive, psychological and social areas.
\end{abstract}

* Máster en Psicología Clínica por la Universidad de Iberoamérica: Licenciada en Psicología por la Universidad de Costa Rica. Actualmente es académica y coordina el Programa "Desarrollo de la Niñez y la Adolescencia" y participa en el programa "Apoyo Psicopedagógico a la Educación Formal y No Formal". en el Instituto de Estudios Interdisciplinarios de la Niñez y la Adolescencia del CIDE. Universidad Nacional.

** Máster en Psicología Industrial y Organizacional por la Universidad Latina de Costa Rica: Licenciada en Psicología por la Universidad de Costa Rica. Actualmente es académica v coordina el Programa "Apoyo Psicopedagógico a la Educación Formal y No Formal" en el Instituto de Estudios Interdisciplinarios de la Niñez y la Adolescencia del CIDE. Universidad Nacional. 
"Había una vez un niño que salía cada día, y el primer objeto que veía, en él se convertía, y este objeto se convertía en parte suya, durante el día o durante parte del día, o por muchos años o ciclos prolongados de años. Las primeras lilas se convirtieron en parte de este niño, y la hierba y las blancas y rojas maravillas, y el rojo y blanco trébol y el canto del pájaro. Y los peces que se mecían tan curiosamente, allá abajo, y el bello líquido curioso, y las plantas acuáticas con sus graciosas cabezas planas, todo esto se convirtió en parte de él."

(Walt Whitman: Leaves of Grass - There was a child went forth, citado por Breckenridge, 1973, pag. 439).

\section{El desarrollo en la infancia}

Para León (1992), el desarrollo del ser humano es quizás una de las manifestaciones vitales más complejas que se conoce, tanto por la multiplicidad de sucesos que se dan simultáneamente, como por la cantidad de factores, internos y externos, que afectan este proceso en sus diferentes etapas.

Así, el desarrollo del ser humano y en particular del niño de 0 a 6 años, que es nuestro interés principal, puede ser figurado como la construcción progresiva de una edificación, en la cual la estructura y las bases se establecen al principio, tal como se da en los primeros seis años de vida del ser humano. Sobre esos pilares se pueden construir muchas variantes, especialmente en esta etapa en que se construyen los cimientos sobre los que se asienta el desarrollo de todo ser humano. Es por este motivo que es importante conocer, estudiar, crear y fomentar las condiciones indispensables para el óptimo desarrollo de cada niño.

Esta autora nos relata que los resultados de estas investigaciones pueden ser utilizados primeramente por los padres, tanto para conocer como para entender y aplicar la información en su proceso de criar a sus hijos. Conociendo las características y habilidades de las diferentes etapas o edades, los padres consiguen ser más realistas en sus exigencias para con sus pequeños, pueden comprender que el niño también tiene sus tareas que cumplir y obstáculos que superar y pueden aceptar la importancia de brindar ciertas condiciones o experiencias en los momentos más propicios para que el menor aprenda. El aplicar estos conocimientos puede permitirle a la familia como tal, un 
funcionamiento más armonioso, pues en el dar y recibir diario, los diferentes miembros de la familia logran ser más comprensivos unos con otros y facilitar en vez de obstaculizar, el desarrollo de cada uno de sus miembros.

Agrega, además, que el desarrollo humano considera todos los procesos y cambios que se dan en el individuo a partir de su concepción y hasta su muerte y que, progresivamente, van formando lo que es el ser humano en los diversos momentos y etapas de su vida. En este proceso intervienen una multiplicidad de factores biológicos y sociales. Sin embargo, existe un tercer factor sumamente importante: la participación activa y no solamente pasiva que tiene el niño y que constituye el componente "individual" del desarrollo. Así, el infante conforme acumula sus experiencias, va formando una estructura mental y física propia y única, diferente de la de los otros pequeños.

Lo anterior conlleva a que, los procesos y cambios que se dan durante el desarrollo pueden ser por lo tanto el producto de la maduración, el crecimiento, el aprendizaje y la experiencia. Conforme avanza, el proceso de desarrollo se vuelve cada vez más complejo y variable.

El desarrollo se da de hecho como un proceso integrado, con ciertas áreas definidas tales como el aspecto físico, emocional, social e intelectual. Este proceso de desarrollo es continuo, pero de acuerdo con el área específica se presentan periodos de aceleración y de latencia. Por ello se considera que existen períodos "críticos" para el desarrollo de ciertas habilidades, funciones u órganos. De esta forma, si se dan las condiciones ambientales necesarias, ya sean internas o externas del individuo, entonces, el proceso se da con más facilidad y más rapidez. Si las condiciones ambientales externas no son favorecedoras o no existen, el proceso se atrasa o se obstaculiza y el desarrollo en general se entorpece (León, 1992).

Es importante subrayar que el desarrollo es un proceso integrado, por lo que los cambios sucedidos en un área del desarrollo repercuten también, en diferentes grados, en el avance de las otras áreas.

\section{Crecimiento y desarrollo en la primera infancia}

La constitución del infante no es estática, sino cambiante. El mismo no vive en un vacío, funciona en un medio ambiente determinado, adquiere experiencias y estas proporcionan un estímulo para desarrollar sus capacidades en formas múltiples. Una gran parte de la estimulación del niño pequeño es de carácter sensible (Caler, 1961, citado por Breckenridge, 1973, p. 4).

Por ello, y para Breckenridge (1973), el menor es la suma de las transacciones de su vida. Se producen cambios a medida que el menor se desarrolla, amplía sus capacidades, tanto físicas como psicológicas y reacciona con madurez creciente a sus experiencias. 
Según Bower (1983), se admite generalmente que la primera infancia. es uno de los períodos más críticos del desarrollo humano. Durante esta etapa se establecen las habilidades cognitivas que llegarán a constituir el intelecto humano. Los pequeños son capaces de muchas cosas que ni siquiera imaginamos y, al mismo tiempo, muestran capacidades que nunca habríamos sospechado. La primera infancia es el momento crítico respecto al desarrollo cognitivo: el período en que pueden tener lugar las adquisiciones y privaciones de mayor importancia. Además. las adquisiciones y privaciones que ocurren en esta etapa son más difíciles de compensar a medida que aumenta la edad.

De esta forma, Gesell (1988), nos amplia agregando que las transformaciones evolutivas que tienen lugar durante el primer año de vida exceden, con mucho. las de cualquier otro período, si se excluyen las del período de gestación. Pero, en el breve lapso de un año, el inerme bebé se yergue sobre sus dos pies y anda, explora y lo escudriña todo. Se ha convertido en un individuo complejo, capaz de emociones diversas, en donde su personalidad y sus aptitudes diversificadas son el producto de una época de crecimiento en extremo veloz.

La clave para comprender la psicología infantil en sus primeros cinco años de vida, se encuentra en su enorme energía, unida a una organización mental de márgenes movibles. Para este autor, es voluble porque la red de neuronas que sustenta el lenguaje florece literalmente con brotes de nuevas adquisiciones, tanto físicas, sociales como mentales.

\section{Estimulación oportuna}

La sociedad es consciente del impacto que tiene el medio ambiente sobre el desarrollo de la personalidad de un individuo, desde el momento mismo de la concepción, pasando por la niñez y juventud, hasta llegar a la vida adulta. Peñaloza (2003) indica que se ha documentado con investigaciones médicas y psicológicas la influencia de un medio ambiente enriquecedor y positivo durante los primeros ocho años de vida en la capacidad de adaptación y el desarrollo de habilidades sociales y cognitivas de las personas, que les permita obtener herramientas adecuadas para la resolución asertiva de problemas cotidianos. Por lo tanto, una de las principales prioridades de los padres, y de la sociedad en pleno, es proporcionar las oportunidades y recursos adecuados para el desarrollo óptimo de los infantes, teniendo en cuenta que la educación y el cuidado de los mismos es una responsabilidad conjunta del hogar, la escuela y la comunidad. 
El término de "estimulación oportuna" se acuña a partir de aportes de diferentes autores tales como Papalia (2001), Hurlock (1990) y Arango, Infante y López (2002) quienes emplean el término de estimulación temprana para referirse a oportuna, e indican que en un principio se llamó estimulación temprana, cuando era todo lo contrario: tardía.

En efecto, la "estimulación temprana" estaba reducida a unas actividades remediales, dirigidas a niños /niñas con severas limitaciones motoras, psíquicas o sensoriales, y que por lo tanto, se situaban en intervenciones con carácter individual y para ser realizadas por personal especializado, en clínicas de atención para aquellos infantes que no lograban alcanzar las metas fijadas para la etapa de desarrollo en el que se ubicaban.

Sin embargo, pronto se evidenció que cuando la terapia era iniciada de forma tardía, los resultados eran pobres por no decir nulos; mientras que cuanto más temprano detectaban las limitaciones, los resultados eran mejores y de mayor alcance. Fue esta la razón por la que le dieron el calificativo de oportuna, para hacer énfasis en la necesidad de iniciarla lo más rápido posible en la vida del infante.

De acuerdo con lo expresado, por Papalia (2001) y Arango et all (2002), lo que en un tiempo se denominó estimulación temprana hoy se llama estimulación oportuna, y ha dejado de ser una terapia remedial, individual y realizada por profesionales para convertirse en una estrategia preventiva, donde se hace partícipe a la comunidad, los distintos miembros de la familia y a las actividades cotidianas en beneficio del desarrollo integral del infante.

\section{¿Qué es la estimulación oportuna?}

La mayor parte de lo que tenemos en la vida, de lo que hacemos cada día es estimulación, desde lo que vemos, lo que escuchamos, lo que gustamos, y en donde usamos los cinco sentidos, puede ser considerado un estímulo, mismo que produce una respuesta en el organismo. A partir de lo anterior, la estimulación oportuna infantil se puede definir como "un acercamiento directo, simple y satisfactorio para ayudar al desarrollo del niño, al tiempo que los padres experimentan gozo y alegría" (Peñaloza, 2003).

Como se mencionó anteriormente, el desarrollo del niño puede afectarse por problemas que se presentan durante el embarazo, durante el parto y después de él, y en los primeros meses de vida tales como desnutrición, ingesta de drogas o alcohol por parte de la madre, infecciones en la misma o del bebé, anomalías genéticas, accidentes en la madre (por ejemplo: caídas, golpes, u otro tipo de 
sufrimiento físico), parto prematuro, falta de oxígeno al nacery ambiente socio-afectivo deficiente (León, 1992; Gesell, 1988, y Bower, 1983).

Parafraseando a León (1992), el desarrollo del infante puede medirse mediante la observación de su conducta la cual se podría dividir en cinco áreas específicas, a saber:

1. Física (involucra la motora gruesa y fina): sus objetivos están orientados para que el niño obtenga un control sobre sus músculos grandes y pequeños, que le permitirán tener la coordinación necesaria para moverse libremente.

2. Lenguaje: se encamina a lograr la comprensión de su lenguaje, para expresarse a través de él.

3. Cognitiva: le permitirá su desarrollo e integración intelectual, a partir de la asimilación, acomodación y adaptación o equilibrio de los estímulos que le brinda el medio ambiente.

4. Psicológica-emocional: se ocupa de hacer al menor independiente, con capacidad para expresar lo que piensa y siente, autónomo y seguro en realizar aquellas tareas que favorezcan la toma de decisiones adecuadas.

5. Social: le proporciona los elementos necesarios para adaptarse al medio ambiente donde se desenvuelve, a partir de la incorporación de normas, valores, principios, creencias, conductas y roles, entre otros.

La adquisición y desarrollo de estas conductas tiene una secuencia lógica que evoluciona acorde con la maduración integral del infante, debido, mayoritariamente al hecho de que todas las actitudes y comportamientos del ser humano están dadas por una secuencia lógica y ordenada, de forma tal que por ejemplo: no podemos esperar que un niño camine si aún no logra sentarse.

Para Woodburn, Boschini y Rodríguez (1997), esta secuencia lógica se puede observar desde el desarrollo motor, en primera instancia, pues este se manifiesta, en el ser humano, mediante la coordinación de músculos, nervios y centros nerviosos, de tal manera que se logre un control de los movimientos del cuerpo. Una de las primera tareas que tendrá el menor conforme avance su crecimiento será la adquisición del control motor.

A criterio de estos autores, aunque no es estrictamente rígido, el patrón que sigue este desarrollo es predecible y está marcado por cinco tendencias:

1. Céfalo caudal: la coordinación y el control ocurren de la cabeza a los pies.

2. Próximo distal: se desarrolla el control de las extremidades distales después del control del tronco. Así se aprende a coordinar el movimiento de los hombros antes que el de los dedos de la mano: y el de la cabeza antes que el de los dedos del pie. 
3. General específico: los patrones motores específicos se desarrollan a partir de movimientos o respuestas generales.

4. Bilateral - unilateral: se desarrolla una preferencia para realizar ciertos movimientos con un lado del cuerpo, previo a un período de experimentación con ambos lados.

5. Músculos gruesos finos: el control de los grupos de músculos gruesos precede al de los músculos finos (pág. 20).

Las habilidades motrices no se desarrollan automáticamente en la vida de infante, ya que no solo dependen de la integridad del aparato motor, sino también de la cantidad y calidad de las experiencias que este tenga con su medio ambiente. Además, estas experiencias que fuerzan la interacción motora del mismo, con el ambiente, son vitales para que él obtenga información por sí solo acerca del medio en que vive.

Añaden que, debido a esta interacción, en general, los menores de 5 años de edad experimentan rápidamente una variedad de habilidades motoras fundamentales. En estas edades el control de los músculos finos aun no se establece, al contrario de los músculos gruesos, los cuales se desarrollan aceleradamente. En términos generales, comienzan a practicar movimientos bilaterales, como galopar, saltar y hacer caballito, que presentan mayor dificultad que los movimientos unilaterales. También se observa un aumento en el dominio del equilibrio.

\section{Finalidad de la estimulación oportuna}

El objetivo de la estimulación oportuna es optimizar el desarrollo del infante para que logre la máxima superación de sus potenciales psicológicos, cognitivos, sociales y físicos, adquiriendo o alcanzando un equilibrio adecuado que permita un desarrollo integrado de su personalidad.

Para Peñaloza (2003), es importante reconocer que no se trata de lograr "niños genio" ni "súper bebés" que puedan ir al baño a los 6 meses o tocar piano a los 2, pues esto es "presionarles" a la satisfacción de necesidades externas (lo que los padres quieren y esperan) más que internas (lo que al bebé le gusta y puede hacer, de acuerdo con el desarrollo neurológico alcanzado).

La estimulación oportuna infantil, debe enfatizar el desarrollo en el ámbito mental, motor, social y emocional por igual, generando comodidad en el mismo y una familiarización de su medio ambiente, como espacio de crecimiento y aprendizaje; creando personas adultas competentes y adaptadas con capacidad de conocer y aprender diferentes tecnologías, de apreciar el arte, de comunicarse con otros y de sentirse satisfechos de ser quienes son. 
lo que en un tiempo se denominó estimulación temprana hoy se llama estimulación oportuna, y ha dejado de ser una terapia remedial, individual y realizada por profesionales para convertirse en una estrategia preventiva, donde se hace partícipe a la comunidad, los distintos miembros de la familia y a las actividades cotidianas en beneficio del desarrollo integral del infante.

\section{¿Qué pueden hacer los padres para mejorar el desarrollo integral de sus hijos e hijas?}

El proporcionar un ambiente seguro y lograr comunicar el amor que sienten por sus hijos, los padres, es la clave para lograr un crecimiento y aprendizaje óptimos. El primer estímulo que recibe el feto de la madre es la respiración: cada minuto la mamá respira de 12 a 16 veces, haciendo expandir el tórax y presionando el abdomen (Peñaloza, 2003).

Esta autora agrega que a la semana 27 de gestación, el feto puede oír los ruidos cardíacos de la mamá, por ello, para estimular el oído del feto pueden hablarle o colocarle melodías cerca del vientre materno. A las 21 semanas de edad gestacional el feto puede recibir estímulos de luz, puede diferenciar cuando las luces están encendidas y cuando están apagadas.

Cuando un niño nace, sus movimientos y actitudes son respuestas reflejas, requiere de estímulos que le darán las experiencias necesarias para el conocimiento y pasar de un estado reflejo (por naturaleza lo realiza) a uno voluntario (controla y busca su conducta).

Si consideramos el tipo de estímulo, el momento en que debe darse, el grado y su calidad, estamos ingresando al mundo de los programas de estimulación oportuna, lo que facilita la potenciación máxima de las posibilidades físicas y mentales del niño, mediante la estimulación continuada y regulada.

No se trata de estimular en forma anárquica (solo como el adulto quiere), sino presentando al niño el mayor número de estímulos posibles, que le agraden y desee repetir; es decir, actividades, basadas en el amplio conocimiento que se debe tener sobre el desarrollo del infante normal en sus diferentes edades, para la promoción de acciones que fomenten y fortalezcan su adelanto.

Para Bassy (1996), esta estimulación depende en gran medida de los objetivos para los que se apliquen. Si se plantean con fines preventivos o terapéuticos, deberán llevarse en instituciones públicas o privadas especializadas en niños, donde participe un equipo compuesto por médicos, terapeutas y 
psicólogos, bajo la coordinación de un especialista en Medicina de Rehabilitación. Sin embargo, si se plantean para el desarrollo de habilidades. o la potenciación de conductas en el infante, pueden ser realizados por el núcleo familiar, o al guna persona significativa en su vida, que posea tiempo y espacio para dedicar a la implementación de guías prácticas simples, pero efectivas en el bebé.

Peñaloza (2003) amplía comentando que debemos tener cuidado con los centros de juego que se autonombran centros de estimulación oportuna y se crearon solo con fines lucrativos, pero que no tienen personal capacitado ni programas con bases científicas.

Si el niño es sano, el mejor programa es el que se aplica en la casa, en un ambiente familiar favorable, en la forma de juego, y bajo la guía de pautas sencillas a aplicar. Para el desarrollo de estas pautas recomendamos observar el suplemento que se adjunta en este artículo, el cual presenta actividades sencillas, útiles y prácticas de realizar con el pequeño según su etapa del desarrollo y área a estimular.

\section{Conclusiones}

La estimulación oportuna es un proceso de integración y potenciación de las habilidades que todo menor de 5 años posee. Es un proceso que se inicia en la familia y que con el tiempo se extiende a todas aquellas situaciones sociales que el pequeño enfrenta como parte de su cotidianeidad.

La estimulación oportuna garantiza una salud adecuada, ayuda a detectar apropiadamente las enfermedades y trastornos del desarrollo para trabajarlos a tiempo. Proporciona, además, una relación afectiva, basada en el contacto físico, el respeto por las necesidades del infante y el establecimiento de límites claros. Es un tipo de manejo preventivo, con bajo costo y fácil de ejecutar; por lo que todo adulto relacionado directamente con un niño debería aprovecharlo y desarrollarlo. Es importante proporcionarle a estos las experiencias necesarias de estimulación, así como altas dosis de cariño, esmero y dedicación.

\section{Referencias}

Arango, M. T.; Infante, E.; y López, M. E. (2002). Estimulación temprana. (Tomos I, II y III). 3ra Edición y 7ma Reimpresión. Colombia: D’vinni Ltda.

Bassy, C. (1996). “La estimulación oportuna”. En: Hacer Familia. (2), 58 -61.

Bower, T. G. R. (1983). El desarrollo del niño/niña pequeño. Madrid: Editorial Debate. 
Bravo, M. Y Pons, L. (2003). "Estimulación oportuna: para que aprendan más y mejor". En: Hacer Familia.(5), 1,66 - 70.

Breckenridge, M. (1973). Crecimiento y desarrollo del niño/niña. México: Editorial Interamericana.

Cerdas, A. I. (1995). Guía didáctica para el desarrollo y aprendizaje en niños de 0 a 3 años. Costa Rica: Ministerio de Educación Pública. - Hall.

Craig, G. (2001). Desarrollo psicológico. (8va Edición). México: Prentice

Gesell, A. (1988). El niño/niña de l a 4 años. España: Editorial Paidós Educador.

Hernández, P. y Rodríguez. A. (1986). Manual operativo para la evaluación y estimulación del crecimiento y desarrollo integral del niño. Costa Rica: Ministerio de Salud.

Hurlock, E.B. (1990). Desarrollo del niño. México: Mc Graw Hill.

León, A. T. (1998). El maestro y los niños: la humanización en el aula. (1 ra Edición). Costa Rica: Editorial de la Universidad de Costa Rica.

León, A.T. (1992). Desarrollo y atención del niño de 0 a 6 años. (2da Edición, primera reimpresión). Costa Rica: Editorial EUNED.

Papalia, D; Wendkos, S. y Duskin, R. (2001). Desarrollo humano. (8va Edición). Colombia: Mc Graw Hill Interamericana, S.A.

Rice, F. (1997). Desarrollo humano: estudio del ciclo vital. (2da Edición). México: Prentice Hall.

Woodburn, S; Boschini, C. y Rodríguez, F. (1997). El desarrollo motor en niños. Costa Rica: Editorial EUNA.

Peñaloza, L. (2003). Documento recuperado en mayo, 2003, del Departamento de Medicina de Rehabilitación del Hospital Infantil de Miami. En: http://www.saludhoy.com 